\title{
Study on Shunt Active Power Filter with Improved Control Method
}

\author{
Yaheng Ren ${ }^{1, a^{*}}$, Xiaozhi Gao ${ }^{2, b}$, Runduo Wang ${ }^{3, c}$ \\ ${ }^{1}$ Institute of Applied Mathematics, Hebei Academy of Sciences, \\ 46 Youyi Street, Shi Jiazhuang, Hebei Province, 050000, PRC \\ ${ }^{2}$ School of Electrical Engineering, Hebei University of Science and Technology \\ 26 Yuxiang Street, Shi Jiazhuang, Hebei Province, 050018, PRC \\ ${ }^{3}$ Automobile and motorcycle industry Development Bureau of Qinghe County \\ Aojie Technology Building, Qinghe County, Hebei Province 054800, PRC \\ ayahengren@126.com, ${ }^{\mathrm{b}}$ gaoxiaozhi@hebust.edu.cn, ${ }^{\mathrm{a}}$ qmjbgs@163.com
}

\begin{abstract}
Keywords - SAPF, improved control method, Ip-Iq current detection method, repetitive control, PI control, harmonic compensation

Abstract.In order to improve power quality and ensure stable operation of power system, shunt active power filter (SAPF) is widely used o filter out harmonics. This paper proposed an improved control method for SAPF. The whole improved control strategy could be divided into two parts: IpIq current detection method to obtain compensation current reference value, and repetitive control combined with PI control to generate PWM pulse to control SAPF to deliver compensation current. The MATLAB simulation results show that SAPF with the proposed control method has good harmonic compensation performance.
\end{abstract}

\section{Introduction}

The wide use of power electronic devices in modern power system has introduced a large number of harmonics, which has caused serious damage to power supply line, power consumption equipments and communication equipments and so on. In order to improve power quality and ensure stable operation of power system, many kinds of measures could be adopted to filter out the harmonics, and filter is the first choice. Filter could be divided into passive filter and active filter. As passive filters may cause resonance, and its filtering effect depends on the system impedance characteristics and is easily influenced by the external conditions, scholars pay more attention on active filter [1]. With advantages of harmonic and reactive power simultaneous compensation, flexible switching and easy control, shunt active power filter (SAPF) is the most widely used active filter device [2][3].

By a certain control method, SAPF detects the harmonic components and then the compensation current is generated in opposite to the harmonic components to achieve the filtering purpose. Therefore, SAPF harmonic compensation performance is mainly determined by the accuracy of the harmonic detection and the accuracy of the current tracking control, which means an effective control method.

However, many scholars did researches on SAPF with different control methods [4]-[9]. Based on the analysis about these references, we can see that there are two common kinds of methods for harmonic detection: p_q method and Ip-Iq method. The active current obtained by p-q method is always distorted when the grid voltage contains distorted and asymmetric components, and the Ip-Iq method is not affected. Therefore, this paper adopted the latter method to detect harmonics .

And also we can see that compensation current control method has been another focus of domestic and foreign scholars. Commonly used technologies are hysteresis current control method and triangular wave modulation control method. And also some artificial intelligence methods are in research. For hysteresis current control method, the current tracking accuracy has been lowered down because of the fixed hysteresis width. For triangular wave modulation control method, the tracking accuracy has been weaken due to harmonics with the same frequency as the triangular carrier. 
Therefore, this paper proposed repetitive control and PI control together to generate PWM pulse to control SAPF to deliver more accurate compensation current.

\section{SAPF working principle}

The schematic diagram of SAPF is shown in Fig.1. The working principle of SAPF is as follows: the current with harmonics is detected first, and then the compensation current reference value is obtained from reference current calculation circuit and as the input to current tracking control circuit, and control signal outputting from current tracking control circuit is to drive the SAPF circuit to deliver the desired compensation current offsetting the harmonics and reactive current eventually.

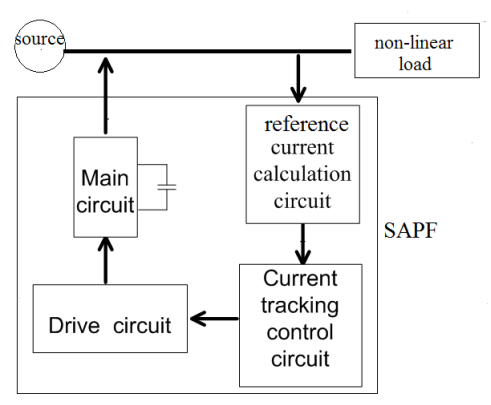

Fig.1. The schematic diagram of SAPF

\section{The improved control method}

The whole improved control strategy could be divided into two parts: Ip-Iq current detection method to obtain compensation current reference value, and repetitive control and PI control together to generate PWM pulse to control SAPF to get compensation current.

A. Ip-Iq current detection method

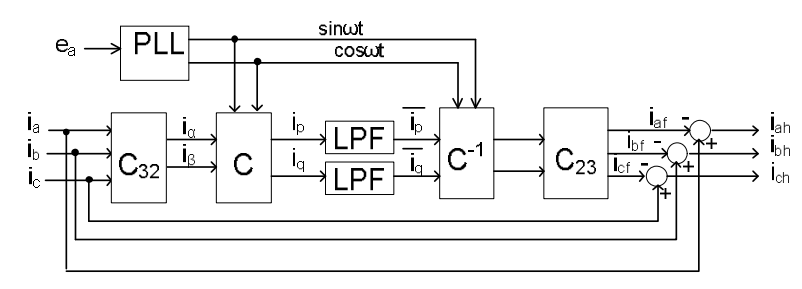

Fig.2. Current detection method

The current detection method is shown as Fig.2 [10].

First, the current detected from non-linear load side is transformed from stationary a-b-c coordinate system to rotating d-q- 0 coordinate system by equation (1) and the current dc values are obtained.

$$
\left[\begin{array}{l}
i_{p} \\
i_{q}
\end{array}\right]=C\left[\begin{array}{l}
i_{\alpha} \\
i_{\beta}
\end{array}\right]=C C_{32}\left[\begin{array}{l}
i_{a} \\
i_{b} \\
i_{c}
\end{array}\right]
$$

Where

$$
\begin{aligned}
& C_{32}=\sqrt{2 / 3}\left[\begin{array}{ccc}
1 & -1 / 2 & -1 / 2 \\
0 & \sqrt{3} / 2 & -\sqrt{3} / 2
\end{array}\right] \\
& C=\left[\begin{array}{cc}
\sin \omega t & \cos \omega t \\
-\cos \omega t & -\sin \omega t
\end{array}\right]
\end{aligned}
$$

And sinwt and coswt are extracted from the phase-locked loop (PLL) segment. 
Then ${ }^{i_{p}}$ and $i_{q}$ are filtered by LPF (Low Pass Filter) respectively to get fundamental current $\bar{i}_{p}$ and $\bar{i}_{q}$... The fundamental value of current in a-b-c coordinate system can be expressed as Equation (2),

$$
\left[\begin{array}{l}
i_{a f} \\
i_{b f} \\
i_{c f}
\end{array}\right]=C_{23}\left[\begin{array}{l}
i_{\alpha f} \\
i_{\beta f}
\end{array}\right]=C_{23} C^{-1}\left[\begin{array}{l}
\bar{i}_{p} \\
\bar{i}_{q}
\end{array}\right]
$$

Where

$$
C_{23}=\left[\begin{array}{cl}
1 & 0 \\
-1 / 2 & \sqrt{3} / 2 \\
-1 / 2 & -\sqrt{3} / 2
\end{array}\right]
$$

Finally the compensation current $\left({ }^{i_{a h}}, i_{b h}\right.$ and ${ }^{i_{c h}}$ ) can be calculated as

$$
\left[\begin{array}{c}
i_{a h} \\
i_{b h} \\
i_{c h}
\end{array}\right]=\left[\begin{array}{l}
i_{a} \\
i_{b} \\
i_{c}
\end{array}\right]-\left[\begin{array}{l}
i_{a f} \\
i_{b f} \\
i_{c f}
\end{array}\right]
$$

Current tracking control--- repetitive control combined with PI control together.

Based on internal model principle, repetitive control has the advantages of improving the system accuracy and realizing zero steady-state error tracking, which makes it a good current control method.

Repetitive control is to correct the original periodic signal by controlling the deviation between periodic signal and feedback signal so as to eliminate the harmonics and then improve the tracking accuracy of the system. The repetitive controller is made up of two parts, the internal module and the compensator, as shown in Fig.3 [10][11].

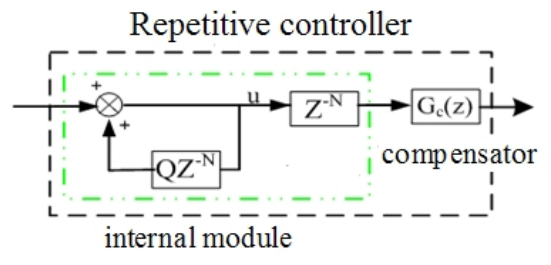

Fig 3. The repetitive controller

The internal module principle is to implant the dynamic model of external signal to controller to form a high precision feedback control system. The value of $Q$ is generally chosen to be a constant less than 1 or a function of a low pass property. In this paper we set $Q$ as 0.95 based on empirical value. The compensator is to offer a control sequence according to the error signal of last cycle and then offset the error quickly and effectively in the next cycle.

Compensator can be expressed as equation (4)

$$
G_{c}(z)=K_{r} \cdot z^{k} \cdot S(z)
$$

Where $\mathrm{K}$ is the gain value for the amplitude compensation of repetitive control, and $K_{r} \in[0,1]$, $z^{k}$ is Lead compensator to eliminate phase lag factor, and $S(z)$ is low pass filter because the gain of repetitive control is small in high frequency section and $\mathrm{S}(\mathrm{s})$ can be expressed as equation (5)

$$
S(s)=\frac{\omega_{n}^{2}}{s^{2}+2 \xi \omega_{n}+\omega_{n}^{2}}
$$

With suitable $\xi$ and $\omega_{n}, \mathrm{~S}(\mathrm{z})$ can be obtained by S(s) discretization.

However, the repetitive controller is a function of the next cycle and has to be combined with better dynamic performance control method in practical application to output signal with steady state performance and quick dynamic response. In this paper, we choose PI control to assist repetitive control. The current tracking control scheme is shown as Fig.4. 


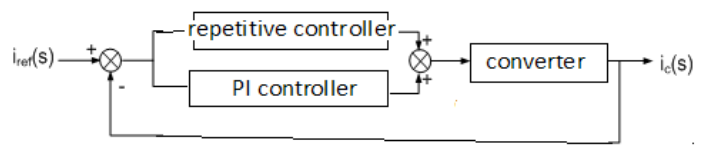

Fig.4 The current tracking control scheme---repetitive control + PI control

According to the practical experience, the formula of proportional-integral part is

$$
\begin{aligned}
k_{p}{ }^{*} & =\frac{L^{*} \omega_{c}}{2 u_{d c}} \\
k_{i}{ }^{*} & =\omega_{c} k_{p}^{*}
\end{aligned}
$$

(6)

Where $\mathrm{L}$ is the total equivalent inductance seeing from the active power filter inverter side to

system side, $\omega_{c}$ is the triangular carrier frequency; $u_{d c}$ is the voltage of SAPF inverter DC side. The working process is: the difference between reference compensation current and SAPF output compensation current is as the input signal for repetitive controller and PI controller, and then the output signals from the two controllers are added together and then modulated by triangular wave to generate PWM signals to control converter and then to make SAPF output compensation current real-time tracking reference current calculated by current detection controlling module. Therefore, SAPF realizes real-time and accurate harmonic compensation.

\section{Simulation Results}

The whole system (Fig.1) is verified in Matlab/Simulink simulation. It is composed of AC power $(220 \mathrm{~V}, 50 \mathrm{~Hz})$, nonlinear load (We choose three-phase bridge rectifier as harmonic source because this kind of equipment is widely used in power system and causes serious harmonic pollution. ) and SAPF with improved control method. Fig.5 is the current waveform of system without SAPF. It can be seen that current contains many harmonic components. If we use FFT to analyze this waveform, we can see that the THD reaches to $14.62 \%$ as shown in Fig.6.

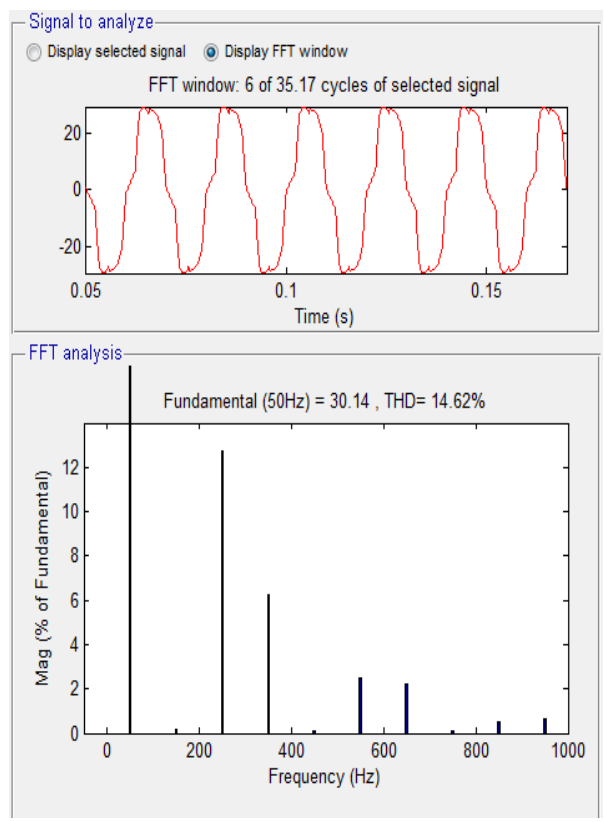

Fig. 5 the current waveform and its THD of system without SAPF

From Fig.6, we can see that the current harmonics have been eliminated by adding SAPF to the system. And the THD of current drops to $2.15 \%$ as shown in Fig.6. 


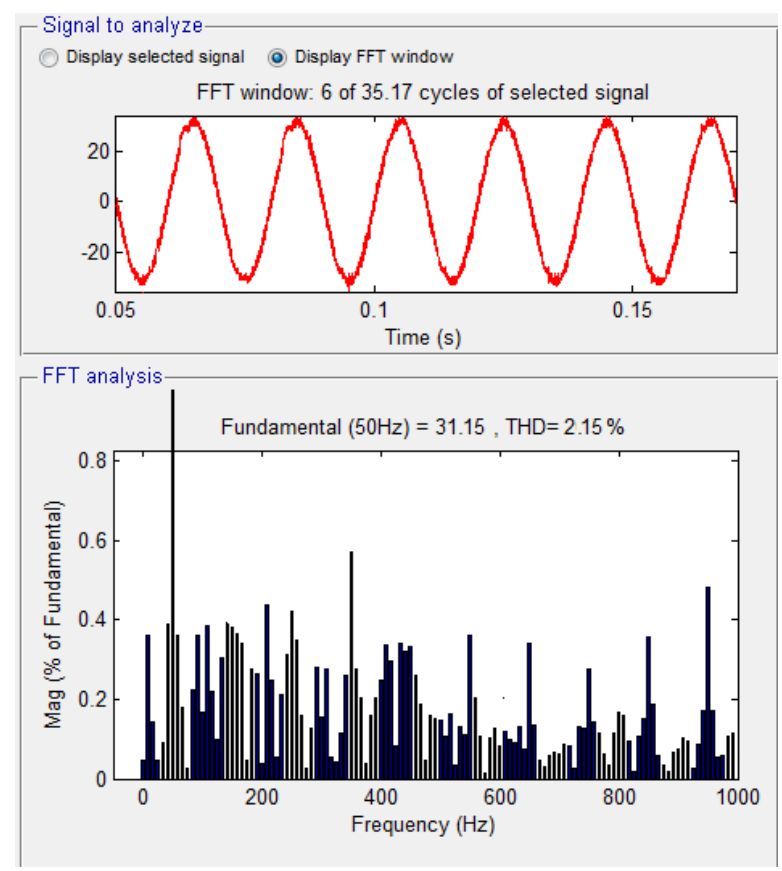

Fig.6 the current waveform and its THD of system withSAPF

To testify the accuracy of the improved control method, power quality improvement results of SAPF with different control methods are shown in Table 1. From Table.1 it can be seen that the lowest THD of current is SAPF with the proposed control method, which means that the proposed method is the most effective among these control methods.

Table1. Power quality improvement results of SAPF with different control methods

\begin{tabular}{|c|c|c|c|c|}
\hline Control methods & $\begin{array}{l}\text { Ip-Iq method } \\
\text { hysteresis current } \\
\text { control method }\end{array}$ & $\begin{array}{l}\text { Ip-Iq method } \\
\text { triangular wave } \\
\text { modulation control } \\
\text { method }\end{array}$ & $\begin{array}{l}\text { the proposed control } \\
\text { method }\end{array}$ & $\begin{array}{l}\text { p_q method } \\
\text { repetitive control + PI } \\
\text { control }\end{array}$ \\
\hline THD & $3.86 \%$ & $4.12 \%$ & $2.15 \%$ & $2.95 \%$ \\
\hline
\end{tabular}

\section{Conclusions}

This paper proposed repetitive control combined with PI control as current tracking control method for SAPF to eliminate harmonic influence in control and improve the current tracking accuracy. Simulation results confirm the effectiveness of the improved control method. Compared with othe control methods, SAPF with the proposed control method shows a better performance in harmonic compensation.

\section{References}

[1]Das J C. Passive filters Potentialities and limitations. IEEE Transaction on Industry Applications, vol. 40(1), pp. 232-241, 2004.

[2]Akagi H, Ogasawara S, Hyosung K. The theory of instantaneous power in three-phase four-wire systems: a comprehensive approach [C]. Proceedings of Industry Applications Conference. New York: IEEE,1999: 431-439.

[3]Bhim Singh, Kamal Al-Haddad, Ambrish Chandra. "A review of active filters for power quality improvement". IEEE Trans, on I.E, vol. 46(5), pp. 1-12,1999

[4] Ucar M, Ozdemir E, Kale M. An analysis of three-phase four-wire active power filter for harmonic elimination reactive power compensation and load balancing under non-ideal mains 
voltage. In proceedings of the 35th Annual IEEE Power electronics Specialists Conference PESC. New York: IEEE, 2004, pp. 3089-3094.

[5] Sun C, Wei G H, Bi Z J. Detection for reactive and harmonic currents of unbalanced three-phase systems based on synchronous reference frame transformation. Proceedings of CSEE, vol. 23(12), pp. 43-48, 2003.

[6]Chen Zhong. Research on the key techniques of shunt active power[D]. Zhejiang University. HangZhou; 2005.

[7 ] Tang Jian, Zou YunPing, MaeLeod Norman. Deadbeat control system with novel neutral- Point balancing scheme for active Power filters. Power Electronics Specialists Conference, 2008

[8] Lianghe Zhu,Yunpin Zou,Xudong Zou, et al. A novel 3-D Space Vector Modulation Scheme for Three-level Three-leg NPC Converter in Three-phase Four-wire APF System. Energy Conversion Congress and Exposition (ECCE), 2012, pp. 1527-1532.

[9]Oleg Vodyakho, Chris C. Mi. Three-Level Inverter-Based Shunt Active Power Filter in ThreePhase Three-Wire and Four-Wire Systems. Power Electronics, IEEE Transactions. Vol. 24 (5).: pp.1350-1363, 2009.

[10]Xiaozhi Gao. Microgrid Control and Power Quality Improvement[D]. Tianjin University. Tianjin,2012

[11] Broberg H, Molyet R G. A new approach to phase cancellation in repetitive control. In proceedings of 1994 Industry Applications Conference 29th IAS Annual Meeting. New York: IEEE, 1994:1766-1770 